\title{
Efficient Test Set Evaluation
}

\author{
Hans-Joachim Wunderlich \\ Maren Warnecke \\ Universität - GHS - Siegen \\ FB 12, Hölderlinstr. 3, 5900 Siegen, Germany
}

\begin{abstract}
The fault coverage obtained by a set of test patterns is usually determined by expensive fault simulation. Even using fault dropping techniques fault simulation provides more information than actually needed. For each fault the pattern is determined which detects this fault first. This is mainly redundant information if diagnosis is not required. We can dispense with this high resolution and restrict our interest on the set of faults which is detected by a set of patterns. It is shown theoretically and practically that this information is obtainable in an highly efficient way.

KEYWORDS: Test, fault simulation

\section{Introduction}

Fault simulation is among the most expensive tasks in circuit design. Its average computing time depends on the size of the circuit, the average size of the list of faults to be simulated and the number of patterns. In summary the average complexity of fault simulation turns out to be between quadratical and cubical [8]. Hence many techniques to improve the efficiency of fault simulation were presented during the last years. They are primarily based on structural analysis to avoid unnecessary computations (e.g. $[10,1,13,12])$ and on Parallel Pattern Single Fault Propagation (PPSFP) for exploiting the parallelism of general purpose computers $[4,17]$.

Compared with classical methods as parallel, deductive or concurrent fault simulation $[14,3,16]$, innovative techniques may be up to 1.000 or 10.000 times faster, but simulating a single pattern still has a quadratical worst case complexity [13]. Complexity analysis shows that there is no hope for linear time fault simulation [9]. Hence many approximate techniques, which are divided into optimistic and pessimistic methods, were proposed to accelerate the evaluation of test sets (e.g. [5, 2, 11]).

For fault $f$ and input pattern $t$ let the detectability $d_{f}(t)$ be computed by an approximate simulator. A method will be called optimistic, if $d_{f}(t)=0$ implies that fault $f$ is not detectable by pattern $t$. It will be called pessimistic, if $d_{f}(t)=$ 1 implies that fault $f$ is detected by pattern $t$. Exact fault simulation is optimistic as well as pessimistic, the "Fast Fault Grading" method proposed by [5] is optimistic, and "Critical Path Tracing" [2] is pessimistic. Unfortunately the latter method has also polynomial complexity with a degree larger than 1 [9].

In the following section we present a technique to compute a pessimistic measure $e_{f}(t)$ and an optimistic measure $\neg \mathrm{n}_{\mathrm{f}}(\mathrm{t})$ for all the faults by a single pass through the cir- cuit with complexity $O(N \ln N)$. Hence $n_{f}(t)=1$ implies that fault $f$ is not detectable by pattern $t$. Another optimistic linear time algorithm for identifying a subset of the undetectable faults was also presented in [Kris90]. Figure 1 illustrates how the set $\mathrm{F}$ of faults is partitioned by the two measures $e_{f}(t)$ and $n_{f}(t)$. As an abbreviation we use the variable $u_{f}(t):=-e_{f}(t) \wedge \neg n_{f}(t)$ for indicating that no information is achieved.

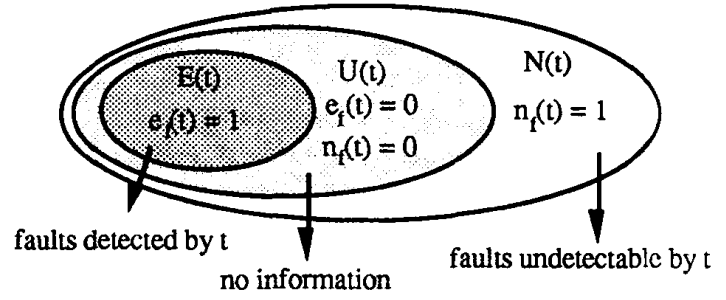

Figure 1: Partition of the fault set $F$

The partition $U(t) \cup N(t) \cup E(t)=F$ can be obtained very efficiently. The algorithm for computing these measures is called Test Set Evaluation (TEVA), and is presented in section 2. In section 3 we explain how to evaluate a sct of test patterns by this algorithm. After applying a number of patterns to TEVA all faults of $E(t)$ can be dropped and we switch to an exact simulator. For the patterns alrcady applied to TEVA we only have to simulate the faults of $\mathrm{U}(\mathrm{t})$, for the following patterns we have to simulate all faults not yet definitely detected. This way we lose the information about the first pattern detecting a fault, but we still retain the exact information about the total fault coverage. Also in this section it is analyzed when to switch from TEVA to exact fault simulation in order to obtain the highest gain in efficiency. Results are presented in section 4.

\section{Approximate Fault Simulation}

The technique presented is based on the principle of single path sensitizing. For each node $k$ and input pattern $t$ two values are computed, which will be called $e_{k}(t)$ and $n_{k}(t)$. $e_{k}(t)$ is true if a single path from $k$ to one of the primary outputs is sensitized. In this case $\mathbf{k}$ is definitely obscrvablc. If $n_{k}(t)$ is true, all pathes from $k$ to the primary oulputs are blocked, and $\mathbf{k}$ is definitely not observable. For all nodes whose values $e_{k}(t)$ and $n_{k}(t)$ are false no statcment can be made.

In order to explain the algorithm in detail, some formal notations are required. Let $\mathrm{C}$ be a combinational circuit 
with primary inputs I and primary outputs $O$, let $v$ be a node of $C$. The boolean function $v^{*}:(0,1)^{I} \rightarrow\{0,1)$ is defined by $v^{*}(t)=1 \Leftrightarrow$ node $v$ becomes logical $" 1$ " if $t$ is applied. The formulas $v^{*}\left(t, 0_{j}\right)$ and $v^{*}\left(t, 1_{j}\right)$ are defined in a similar way, but in addition node $i$ is set to 0 or 1 , respectively. If $\mathrm{f}$ is a stuck-at fault $\mathrm{s} 0-\mathrm{k}$ [or $\mathrm{s} 1-\mathrm{k}$ ] we define $e_{f}(t)=k^{*}(t) \wedge e_{k}(t)\left[e_{f}(t)=\neg k^{*}(t) \wedge e_{k}(t)\right]$ and $n_{f}(t)$ $=\neg k^{*}(t) \vee n_{k}(t)\left[n_{f}(t)=k^{*}(t) \vee n_{k}(t)\right]$. Hence $e_{f}(t)=1$ implies definite fault detection, and $n_{f}(t)=1$ implies that fault $f$ is undetectable.

Let $\mathbf{k}$ be a node with the immediate predecessor $\mathbf{v}$. The term $\frac{\delta k}{\delta v}(t)$ describes the boolean difference, it is true if the value of $v$ can be observed at $k$. In figure 2 we have $\frac{\delta \mathrm{g}}{\delta \mathrm{k}}=1$, and $\frac{\delta \mathrm{g}}{\delta \mathrm{b}}=0$.

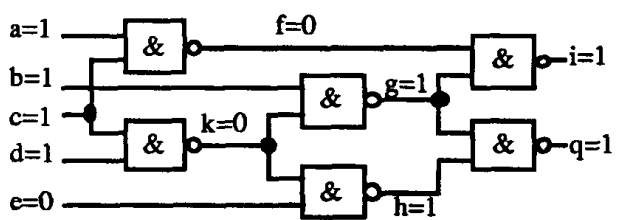

Figure 2: $\quad$ Example circuit $\mathrm{C} 17$

As combinational circuits can be considered as acyclic graphs, a path from node $v$ to a node $o$ is uniquely determined by its set of nodes $\omega(v, 0)$. If $\omega(v, k)$ is a path from $v$ to $k$ and $\omega(k, 0)$ is a path from $k$ to $o$, the formula $\omega(v, k)+\omega(k, 0)$ denotes the well-defined path from $v$ to $o$ formed by the concatenation of $\omega(v, k)$ and $\omega(k, 0)$.

Lemma 1: Node $v$ is not observable if for each output $o \in O$ and for each path $\omega(v, 0)$ there is a node $z \in \omega(v$, o) such that $z^{*}\left(t, l_{v}\right)=z^{*}(t, 0 v)$.

Proof: Straightforward

A path $\omega(\mathrm{v}, 0)$ is called blocked by node $\mathrm{v}_{\mathrm{i}+1} \in \omega(\mathrm{v}, 0)$ if $v_{i}$ is not observable at $v_{i+1}$, and all the other immediate predecessors $k \neq v_{i}$ of $v_{i+1}$ are independent of $v, i$. e. $k^{*}(t$, $\left.0_{v}\right)=k^{*}(t, 1 v)$.

Without loss of generality we assume a maximum fanout of 2 in the circuit. With the help of the following definition we can decide whether a fanout stem is definitely not observable:

Definition 1: Let $\mathrm{g}, \mathrm{h}$ be two immediate successors of $v$. The two node sets $\mathrm{A}_{1}, \mathrm{~A}_{2}$ satisfy the blocking condition of $\mathrm{v}$ if:

1) For each output $o$ and path $\omega_{1}(\mathrm{~g}, 0)$ there is a blocking node in $A_{1}$.

2) For each output 0 and path $\omega_{2}(\mathrm{~h}, 0)$ there is a blocking node in $\mathbf{A}_{2}$.

3) For every $b \in A_{1} \cap A_{2}$, the immediate predecessor of $b$ in $\omega_{1}(g, 0)$ is also predecessor in $\omega_{2}(h, 0)$.
Obviously the blocking condition is satisfied for $A_{1} \cap A=$ $\varnothing$. In figure $3 a)$ the path $\omega_{1}(g, 0)$ is blocked by $A_{1}:=\{0\}$ and $\omega_{2}(h, 0)$ is blocked by $A_{2}:=\{0\}$, too, but the blocking condition is violated due to 3 ), so that we cannot make any statement about the observability of $k$. a)

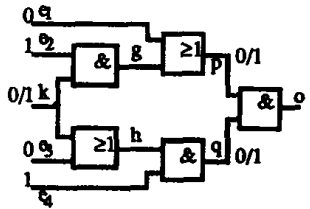

b)

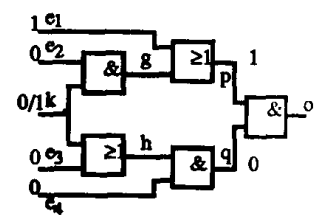

Figure 3: Blocking condition

In figure $3 b$ ) we have $A_{1}:=\{p\}$ and $A_{2}:=\{q\}$, i.e the blocking condition is satisfied and $\mathbf{k}$ is definitely not observable.

Lemma 2i If $A_{1}, A_{2}$ satisfy the blocking condition for $v$ then the set $A_{1} \cup A_{2}$ contains blocking nodes for all paths $\omega(v, 0)$ and $v$ is not observable.

Proof: $\quad$ see [18], p. 170.

Now we can derive a sufficient condition of definite obscrvability. Under patter $t \in\{0,1\}^{I}$, a single path $\omega(v, w)$ $:=\left\{v=v_{0}, \ldots, v_{n}=w\right\}$ is sensitized if

a) All nodes of $\omega(v, w)$ have different values for $v=1$ and $v=0: v_{i}^{*}(t, 0 v) \neq v_{i}^{*}\left(t, 1_{v}\right), i=0, \ldots, n$.

b) All immediate predecessors $k \neq v_{i-1}$ of $v_{i}, i=1, \ldots$, $\mathrm{n}$, outside the path have the same value for $\mathrm{v}=1$ and $v=0$, i.e. $k^{*}\left(t, 0_{v}\right)=k^{*}\left(t, 1_{v}\right)$.

Figure 4 illustrates the concept of single path sensitizing:

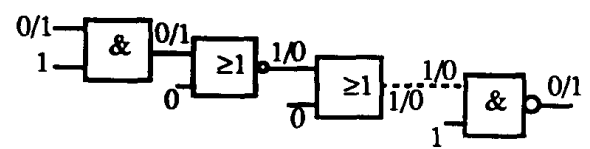

Figure 4: Single path sensitizing

If a single path from node $\mathrm{v}$ to a primary output is scnsitized then $v$ is observable, but this conclusion is not always ture in the other direction. Finally we have:

Lemm? 3: Let $v$ be a fanout stem with the immcdiate successors $g$ and $h, i$. e. there are edges $e_{1}:=(v, g)$ and $e_{2}:=(v, h)$. Let a path $\omega(g, 0)$ be sensitized and let $\frac{\delta g}{\delta v}=$ 1. Let $N\left[e_{1}\right]$ be a set of nodes such that for each path $\omega_{1}:=(v, g, \ldots, p) \not \subset(v, g)+\omega(g, o)$ with some $p \in$ $\omega(g, o)$ there is a blocking node $b \in N\left[e_{1}\right], b \neq p$. Let $\mathrm{N}\left[e_{2}\right]$ be analogously defined for all paths $(v, h, \ldots, 0)$. If $N\left[e_{1}\right] \cap N\left[e_{2}\right]=\varnothing$ and $N\left[e_{2}\right] \cap \omega(g, o)=\varnothing$ then $(v, g)$ $+\omega(\mathrm{g}, 0)$ is a sensitized single path. Additionally $N[\mathrm{v}]:=$ $N\left[e_{1}\right] \cup N\left[e_{2}\right]$ contains blocking nodes for all paths $\tau(v$, p) $\varnothing(\mathrm{v}, \mathrm{g})+\omega(\mathrm{g}, \mathrm{o})$ with some $\mathrm{p} \in(\mathrm{v}, \mathrm{g})+\omega(\mathrm{g}, \mathrm{o})$. Proof: see [18], p. 170. 
Lemma 3 gives us a means for deciding whether a single path starting from a fanout stem is sensitized. The complexity of this decision is in the order of the cardinalities of the sets $\mathbf{N}$.

We clarify lemma 3 with the help of the example circuit of figure 2. If we want to compute the observability of the fanout stem $c$, the two sets $N\left[e_{1}\right]\left(e_{1}:=c, f\right)$ and $N\left[e_{2}\right]$ $\left(e_{2}:=c, k\right)$ have to be determined. We set $N\left[e_{1}\right]:=\varnothing$ and $N\left[e_{2}\right]:=\{h, i\}$, and the path $\omega(f, i)$ is sensitized. Because of $N\left[e_{2}\right] \cap \omega(f, i) \neq \emptyset$, the condition of single path sensitizing is not satisfied and we cannot make any statement about the observability of $\mathbf{k}$.

The approximate simulation algorithm TEVA consists of two steps. First the fanout-free regions are processed by finding out if the path from node $k$ to the next fanout stem or primary output, which is called $\mathbf{v}(\mathbf{k})$, is sensitized. In this case $e^{\prime} k$ is true; if $k$ is a fanout stem or primary output by itself, i. e. $k=v(k)$, formula $e_{k}^{\prime}$ is true, too.

Case 1) $\quad v(k)=k$ : $\quad$ Set $e_{k}^{\prime}=1$.

Case 2) $\quad v(k) \neq k: \quad$ Set $e_{k}^{\prime}=\frac{\delta k 2}{\delta k} \wedge e_{k 2}^{\prime}$, where $k 2$ is the successor of $k$.

During the second step the whole circuit is processed by calculating $e_{k}$ and $n_{k}$ for each node. Whithin fanout-free regions and for primary outputs this is straightforward:

Case 1) $\quad k$ is a primary output: Set $e_{k}=1 ; n_{k}=0$.

Case 2) $\quad k$ is inside a fanout-free region: Set $e_{k}=e_{k}^{\prime} \wedge e_{v(k)} ; n_{k}=\neg e_{k}^{\prime} \vee n_{v(k)}$.

Case 3)

In order to compute $e_{k}$ and $n_{k}$ for fanout stems we have to determine the already introduced set $N[k]$ which contains the blocking nodes and a set called $S[k]$. If $e_{k}$ is true, $S[k]$ will contain all the nodes of the sensitized path from $k$ to a primary output of the circuit, otherwise $S[k]$ is empty. The successors of fanout stems are divided into independent and dependent successors. If there are reconvergent paths to the same primary output, they are called dependent and we apply lemma 3. Otherwise there are no reconvergencies and all paths starting at the two successors lead to disjoint primary outputs.

Before processing fanout stem $\mathbf{k}$, we first have to determine the two values $\mathrm{e}$ and $\mathrm{n}$ and the set $\mathrm{N}$ for the two edges leading from that fanout stem to his immediate successors $g$ and $h$ : We define $e_{g, k}=\frac{\delta g}{\delta k} \wedge e_{g}$ and $n_{g, k}=\neg \frac{\delta g}{\delta k}$ $\vee n_{g}$. If $\left(e^{\prime} g \wedge \frac{\delta g}{\delta k}\right)=0$ we set $N[k, g]:=\left\{k^{\prime}\right\}$, where $k^{\prime}$ is the node of path $\omega(\mathrm{g}, \mathrm{v}(\mathrm{g}))$ nearest to $k$, else we set $\mathrm{N}[\mathrm{k}, \mathrm{g}]:=\mathrm{N}[\mathrm{v}(\mathrm{g})]$.

After processing the edge $(k, h)$ the same way, we can determine the observability of $\mathrm{k}$ as shown in figure 5 .

\subsection{Complexity and accelerations}

The algorithm consists of two passes through the circuit.
Except for fanout stems the number of operations at a node is bounded by a constant. At fanout stems we have to intersect sets which increase linearly with the circuit size C. Overall this leads to a quadratical worst-case complexity, which can be reduced further.

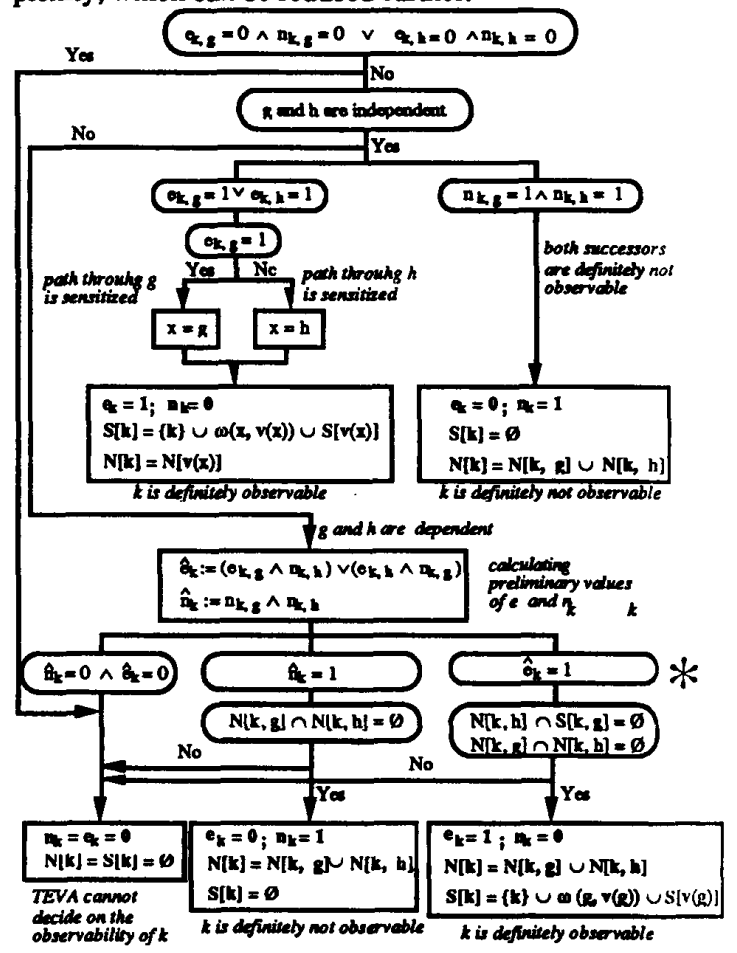

Figure 5: Determining the observability of fanout stcms

* The path through $\mathrm{g}$ is sensitized. If the path through $h$ is sensitized, $e_{k}, n_{k}, N[k]$ and $S[k]$ are computed the analogous way.

As the sets S represent paths of linearly ordered nodes they can be organized as balanced trees such that checking membership can be done in $\mathrm{O}(\mathrm{ln}|\mathrm{S}|)$. Moreover the tradeoff between the information obtained by this algorithm and its complexity can be controlled by an additional parameter $\beta$ which should limit the cardinality of the sets $N$. Whenever IN[k, g] $\cup N[k, h] l>\beta$ we dispense with further computations for $k$, and set $N[k]=S[k]=\emptyset$ and $n_{k}=$ $e_{k}=0$. With this restriction the entire algorithm has complexity $\mathrm{O}(\mathrm{C} \cdot \ln (\mathrm{C})$ ). Further improvements are obtainable by an analysis of the circuit structure as used in exact fault simulation [MaRa88, Schu88]. E. g. if node $k$ has a dominator $d$ the computation can be accelerated [15]. Suppose $\mathbf{d}$ is definitely not observable, then $\mathbf{k}$ is not ob. servable either. We set $n_{k}=1, s_{k}=0, N[k]=N[d]$, and $\mathbf{S}[\mathbf{k}]=\varnothing$.

Finally the principle of Parallel Pattern Single Fault Propagation $[4,17]$ can be applied to all steps of the algorithm with the exception of the set operations. The results 
to be reported in section 4 are obtained by the approximate method and by an exact fault simulation both using the PPSFP priciple and a preprocessing task for determining dominators.

\section{Evaluation of large test sets}

The improvements of efficiency obtained by the approximate method increase with the number of faults to be processed. If the fault list is reduced by fault dropping the exact simulation may become even faster. This section addresses the problem of balancing the computational load between the approximate method and the exact fault simulation. A similar problem was dealt with in [7] where the best point for switching from random pattern simulation to deterministic test generation was discussed.

Using TEVA the fault coverage obtained by the test set $T$ $:=\left\{t_{1}, \ldots, t_{\alpha}\right\}$ is determined in the following way:

1) Set $F_{0}:=F$ and for all $f \in F$ set $\overline{T(f)}:=\varnothing$

2) For $i=1, \ldots, \alpha:$ Set $\hat{F}_{i}:=$ the set of faults in $F_{i-1}$ detected by $t_{i}$ using TEVA. Set $F_{i}:=F_{i-1} \backslash \hat{F}_{i}$.If TEVA classifies fault $f \in F_{i}$ as not detectable by pattern $t_{i}\left(i\right.$. e. $\left.n_{f}\left(t_{i}\right)=1\right)$ then set $\overline{T(f)}:=\overline{T(f)} \cup\left(t_{i}\right)$.

3) Do exact fault simulation for all faults $f \in F_{\alpha}$ with patterns from $T \backslash \overline{T(f)}$.

First we determine the expected number of patterns and the expected computational effort for detecting a fault by exact simulation, then the same values are determined for TEVA. Finally a switching criterion is established and the possible savings are quantified.

Let cs: Characteristic constant of the complexity of the exact fault simulation method used.

C: Size of the circuit.

$\alpha$ : Number of test patterns to be evaluated.

Let $d_{f}, e_{f}, n_{f}$ and $u_{f}$ be the probability that $d_{f}(t), e_{f}(t), n_{f}(t)$ and $u_{f}(t)$, respectively, are true if $t$ is a random pattern. The expected number of random patterns until detecting and dropping fault $f$ is described by the formula

(1) $E(f)=\sum_{i=1}^{k} i\left(1-d_{f}\right)^{i-1} \cdot d_{f}$.

Some simple transformations yield

$$
E(f)=-d_{f} \frac{\delta \sum_{i=1}^{\alpha}\left(1-d_{f}\right)^{i}}{\delta d_{f}}=\frac{1-\left(1-d_{f}\right)^{\alpha}}{d_{f}}-\alpha\left(1-d_{f}\right)^{\alpha} \text {. }
$$

The probability of not detecting a fault during simulation is $\left(1-d_{f}\right)^{\alpha}$, and $\alpha \cdot\left(1-d_{f}\right)^{\alpha}$ patterns are expected. Hence on the average

(3) $\quad a(f):=E(f)+\alpha\left(1-d_{f}\right) \alpha:=\frac{1-\left(1-d_{f}\right) \alpha}{d_{f}}$

patterns have to be simulated for fault $f$, and the expected simulation costs are

(4) $c_{S} \cdot \sum_{f \in F} a(f) \cdot C$.

For estimating the total effort for the combination of approximate and exact fault simulation the following parameters are used

$\hat{\mathbf{e}}_{\mathrm{f}}: \frac{\mathrm{e}_{\mathrm{f}}}{\mathrm{d}_{\mathrm{f}}}$ describes the quality of the pessimistic approximation of TEVA

$\hat{n}_{f}: \frac{n_{f}}{1-d_{f}}$ describes the quality of the optimistic approximation of TEVA

$\alpha_{\mathrm{f}} \quad:\left(1-\mathrm{n}_{\mathrm{f}}\right) \boldsymbol{\alpha}$ expected number of patterns $\mathrm{T} \backslash \overline{\mathrm{T}(\mathrm{f})}$ to be simulated

$\tilde{\mathrm{d}}_{\mathrm{f}}: \frac{\mathrm{d}_{\mathrm{f}}}{1-\mathrm{n}_{\mathrm{f}}}$ is the conditional detecting probability if a pattern from $T \backslash \overline{T(f)}$ is simulated.

$\mathrm{C}_{\mathrm{T}}(\beta)$ the characteristic constant of the complexity of TEVA using parameter $\beta$.

As the effect of fault dropping on the computational effort of TEVA is negligible step 2 leads to an worst casc cffort of order

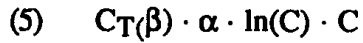

The probability of a fault not being detected in Step 2) is $\left(1-e_{f}\right)^{\alpha}$. Hence in step 3 we expect a simulation time in the order of

$c_{S} \cdot \sum_{\mathbf{f} \in \mathrm{F}} \mathrm{a}^{\prime}(\mathrm{f}) \mathrm{C}$ where

$a^{\prime}(f)=\left(1-e_{f}\right)^{\alpha} \frac{1-\left(1-\tilde{d}_{f}\right)^{\alpha_{f}}}{\tilde{d}_{f}}$.

Due to the power series of the exponential function we estimate $\left(1-e_{f}\right)^{\alpha}=\left(1-\hat{e}_{f} \cdot d_{f}\right) \alpha \approx\left(1-d_{f}\right) \hat{e}_{f} \alpha$, and

$\frac{1-\left(1-\tilde{d_{f}}\right)^{\alpha_{f}}}{\tilde{d_{f}}}=\frac{1-\left(1-\frac{d_{f}}{1-n_{f}}\right) \alpha_{f}}{\frac{d_{f}}{1-n_{f}}} \approx \frac{1-\left(1-d_{f} ;\right.}{\frac{d_{f}}{1-n_{f}}}$,

and hence we have

$$
a^{\prime}(f)=\left(1-d_{f}\right) \hat{e}_{f} \alpha\left(1-\hat{n}_{f}\left(1-d_{f}\right)\right) \frac{1-\left(1-d_{f}\right)^{\alpha}}{d_{f}} .
$$

The costs of simulating fault $f$ are $a(f)=\frac{1-\left(1-d_{f}\right) \alpha}{d_{f}}$, the costs of simulating fault $f$ after TEVA are $a^{\prime}(f)$ which is only a fraction:

(6) $\frac{a^{\prime}(f)}{a(f)}=\left(1-d_{f}\right) \hat{e}_{f} \alpha \cdot\left(1-\hat{n}_{f}\left(1-d_{f}\right)\right)$

The results reported in section 4 show average valucs for $\hat{\mathrm{e}}_{\mathrm{f}}$ significantly larger than 0.5 and for $\hat{\mathrm{f}}_{\mathrm{f}}$ significantly larger than 0.9 . Hence the fraction (6) is very small, its 
exact size depends on the actual detectibility $d_{f}$, too.

In general it is not useful to process all the patterns by TEVA as after reducing the fault list by fault dropping TEVA loses and exact simulation gains efficiency. The optimal switching point is determined as described in [7].

Assume after applying i patterns the subset $\tilde{F}$ of faults is still undetected by TEVA. Then the expectation value for each $e_{f}(t), f \in \widetilde{F}$, is $E\left(e_{f}\right)=\frac{1}{i+2}$ [7].

An estimation of the portion of faults not detectable by TEVA but by simulation is $1-\hat{e}$, where $\hat{e}:=\frac{1}{|F|} \sum \hat{e}_{f \in F}$ is the average quality of TEVA. Hence by applying another pattern to TEVA we expect an increase of fault coverage of at least $\Delta \mathrm{T}:=(\tilde{\mathrm{F}}|-(1-\hat{\mathrm{e}})| \mathrm{F} \mid) \frac{1}{i+2}$, until $|\tilde{F}|>(1-\hat{e})|F|$. By applying another pattern to exact simulation we expect an increase of fault coverage by

$$
\Delta \mathrm{S}:=\tilde{\mathrm{F}}\left|\cdot \mathrm{E}\left(\mathrm{p}_{\mathrm{f}}\right)=\tilde{\mid \mathrm{F}}\right| \cdot \frac{1}{\hat{\mathrm{e}}} \mathrm{E}\left(\mathrm{e}_{\mathrm{f}}\right)=\frac{|\tilde{\mathrm{F}}|}{\hat{\mathrm{e}}(\mathrm{i}+2)} .
$$

The costs of fault coverage in time units determine the switching point: Using formula (4) and (5), if

$$
\Delta_{\mathrm{S}} \cdot \frac{1}{\mathrm{C}_{\mathrm{S}}|\tilde{\mathrm{F}}|}>\Delta_{\mathrm{T}} \cdot \frac{1}{\mathrm{C}_{\mathrm{T}}(\beta) \ln (\mathrm{C})}
$$

then exact simulation becomes more efficient than TEVA. As $\Delta_{\mathrm{S}}$ and $\Delta_{\mathrm{T}}$ are only estimations the switching point should not be precomputed statically but checked dynamically. If the expected gain $\Delta \mathrm{T}$ in fault coverage is not reached for several times, we should dispense with TEVA and switch to exact simulation. On the other hand, if the actual increase $\Delta_{T}$ in fault coverage exceeds we continue with TEVA.

\section{Experimental results}

The usefulness of the presented approach depends on the answers of two questions. First we have to examine if the estimated values are sufficiently precise, i. e. if the average value of $u_{f}$ is small enough. Second we investigate whether the approximate technique is fast enough in order to achieve an improvement of performance for the combination of exact and approximate simulation. It will be shown that both questions can be positively answered for the large combinational and sequential ISCAS-benchmark circuits [Brgl85, 89]. Only the combinational parts of the sequential circuits are used.

In order to evaluate the average quality of the pessimistic and the optimistic approximation of TEVA, each circuit was simulated with a test set $T$ of 6000 pattern by both techniques. For each pattern $t$ we determined $d_{f}(t)$, $e_{f}(t)$, $n_{f}(t)$ and $u_{f}(t):=1-e_{f}(t)-n_{f}(t)$. The average quality of the pessimistic and the optimistic estimation is

$$
\hat{\mathrm{e}}:=\frac{1}{|\mathrm{~F}|} \sum_{\mathrm{f} \in \mathrm{F}} \hat{\mathrm{e}}_{\mathrm{f}} \text {, and } \hat{\mathrm{n}}:=\frac{1}{|\mathrm{~F}|} \sum_{\mathrm{f} \in \mathrm{F}} \hat{\mathrm{n}}_{\mathrm{f}}
$$

The average degree of uncertainty is $u:=\frac{1}{|F|} \sum_{f \in F} \frac{\sum_{1 \in T}}{|T|}$.

The values of $d, e$ and $n$ are computed the same way.

Table 2 contains these experimentally determined valucs, which demonstrates the quality of TEVA.

\begin{tabular}{|l|l|l|l|}
\hline circuit & $\mathbf{u}$ & $\hat{\mathrm{e}}$ & $\hat{\mathbf{n}}$ \\
\hline C1908 & 0.156 & 0.514 & 0.913 \\
\hline C2670 & 0.185 & 0.294 & 0.908 \\
\hline C3540 & 0.156 & 0.086 & 0.929 \\
\hline C5315 & 0.189 & 0.229 & 0.886 \\
\hline C6288 & 0.254 & 0.096 & 1 \\
\hline C7552 & 0.171 & 0.324 & 0.977 \\
\hline S9234 & 0.116 & 0.612 & 0.933 \\
\hline S13207 & 0.099 & 0.649 & 0.962 \\
\hline S15850 & 0.111 & 0.584 & 0.949 \\
\hline S35932 & 0.151 & 0.545 & 0.897 \\
\hline S38584 & 0.078 & 0.744 & 0.967 \\
\hline S38417 & 0.113 & 0.588 & 0.945 \\
\hline
\end{tabular}

Quality of the estimations

The quality of the pessimistic approximation increases with the circuit size and is for the six larger circuits on the average $\mathbf{0 . 6 2}$. The average quality of the optimistic approximation is $\mathbf{0 . 9 4 2}$. The quality of the approximate method also depends on the circuit structure, e.g. the quality of the estimations for circuit C6288 is much worse than for the other circuits. This circuit has a large portion of lanout stems, each of these fanout stems has dependent succcssors. In contrast to this, the quality of the approximations for S38584 is very high. This circuit has less fanout stems than S35932 and S38417. A lot of these fanout stems have only independent successors.

Table 3 shows that the high quality estimations are obtained faster than exact simulation can be performed, and that TEVA depends linearly on the circuit size.

\begin{tabular}{|l|l|l|l|l|l|}
\hline & \multicolumn{2}{|c|}{ TEVA } & \multicolumn{2}{c|}{ SLM } & \\
\hline circuit & time & $\frac{\text { CPU-ms }}{\text { node }}$ & time & $\frac{\text { CPU-m }}{\text { node }}$ & $\frac{\text { timcSIM }}{\text { iimeTIV }}$ \\
\hline C1908 & 0.6 & 6.6 & 1.3 & 13.9 & 2.1 \\
\hline C2670 & 0.4 & 2.6 & 1.4 & 9.8 & 3.8 \\
\hline C3540 & 0.8 & 4.8 & 3.5 & 20.3 & 4.2 \\
\hline C5315 & 0.6 & 2.4 & 4.2 & 17.0 & 7.0 \\
\hline C6288 & 0.9 & 3.5 & 14.3 & 58.4 & 16.7 \\
\hline C7552 & 1.5 & 4.0 & 11.8 & 31.6 & 7.8 \\
\hline S9234 & 1.6 & 2.7 & 13.2 & 22.6 & 8.3 \\
\hline S13207 & 2.0 & 2.3 & 17.8 & 20.6 & 8.9 \\
\hline S15850 & 2.4 & 2.4 & 30.7 & 29.0 & 12.7 \\
\hline S35932 & 5.8 & 3.3 & 184.5 & 103.5 & 32.2 \\
\hline S38584 & 8.2 & 3.5 & 214.0 & 89.7 & 26.3 \\
\hline S38417 & 6.6 & 3.2 & 180.2 & 87.0 & 27.0 \\
\hline
\end{tabular}

Table 3: Computing times in CPU-s for simulating 32 patterns

The last column is the ratio of timesIM and time TEVA 
which increases with the circuit size. The preprocessing task is the same for both techniques, has to be performed only once and is not taken into account. All results of TEVA are obtained on a SPARC 1+ workstation using the parameter $\beta=5$.

The complexity constants $C_{S}$ and $C_{T}(\beta)$ are determined experimentally using quadratical minimization techniques and we get $C_{S}=9.73 \cdot 10^{-9}$ and $C_{T}(5)=1.02 \cdot 10^{-6}$.

Table 4 contains the fault coverage $F_{S}$ where it is useful to switch from TEVA to exact simulation. $\alpha_{S}$ is the number of patterns simulated by TEVA before switching to exact simulation.Moreover it shows the time for the exact simulation and the fault coverage obtained by SIM.

\begin{tabular}{|l|l|l|c|c|c|}
\hline & & \multicolumn{2}{|c|}{ TEVA } & \multicolumn{2}{c|}{ SIM } \\
\hline circuit & $\alpha S$ & time & FS & time & F \\
\hline S9234 & 928 & 63.8 & 0.36 & 199.1 & 0.69 \\
\hline S13207 & 1312 & 153.0 & 0.50 & 365.1 & 0.74 \\
\hline S15850 & 576 & 67.4 & 0.47 & 268.5 & 0.79 \\
\hline S35932 & 160 & 51.0 & 0.47 & 289.3 & 0.90 \\
\hline S38584 & 4046 & 1444.6 & 0.68 & 2628.3 & 0.84 \\
\hline S38417 & 576 & 210.8 & 0.48 & 1087.5 & 0.92 \\
\hline
\end{tabular}

Table 4: Fault coverages and CPU-times in $s$ for exact simulation and TEVA (simulating $\alpha_{S}$ patterns)

TEVA requires computing times 1.5 through 5 times less than exact simulation in order to determine the detection of $52 \%$ through $81 \%$ of the detectable faults. Computing time is still saved if TEVA and exact simulation are combined. The results of simulating $\alpha_{S}$ patterns with TEVA and than simulating the following patterns with the exact simulator are shown in table 5. The combination of TEVA and exact simulation described in section 3 could not be examined because at the current stage of the implementation of the exact fault simulator it is not possible to eliminate those patterns where $n_{f}(t)=1$. In order to exploit the additional information of the optimistic approximation obtained by TEVA the exact simulator will be modified.

\begin{tabular}{|l|l|l|l|l|}
\hline & \multicolumn{2}{|c|}{ simulation } & \multicolumn{2}{c|}{ TEVA+simulation } \\
\hline circuit & F & time & F & time \\
\hline S9234 & 0.7349 & 325.7 & 0.712 & 245.9 \\
\hline S13207 & 0.8133 & 571.7 & 0.8016 & 458.0 \\
\hline S15850 & 0.8395 & 413.4 & 0.8132 & 249.9 \\
\hline S35932 & 0.8959 & 426.87 & 0.8959 & 326.9 \\
\hline S38584 & 0.9311 & 4218.6 & 0.9256 & 4071.4 \\
\hline S38417 & 0.8616 & 1757.2 & 0.8525 & 1143.4 \\
\hline
\end{tabular}

Table 5: Fault coverages and computation time in CPU-s for the exact simulation and combining TEVA with simulation for $2 \alpha_{S}$ pattern

Experimental results have demonstrated that the choice of the parameter $\beta$, which limits the cardinality of the sets $N$, has only small influence on the accuracy of the results. For all of the examined circuits the fault coverage is nearly constant - independent of the value we assign to $\beta$. Since the parameter primarily influences the computation time and not the accuracy of the simulation results, the value 5 for $\beta$ seems to be a good choice.

\section{Conclusion}

An efficient method for evaluating the fault coverage obtained by large test sets has been presented. With nearly linear effort it is determined whether a certain pattern definitely detects a fault or is unable to detect or whether no information is obtainable by this method. Only for the latter case exact simulation is required. Overall this lcads to a significant reduction of computing time.

\section{References}

1 Antreich, K.J.; Schulz, M.H.: Accelerated Fault Simulation and Fault Grading in Combinational Circuits; IEEE Trans. CAD, Vol. CAD-6, No. 5, 1987, pp. 704-712

2 Abramovici, M.et al.: Critical Path Tracing - An Alternative to Fault Simulation; Proc. of 20th Design Automation Conference, 1983, pp. 214-220

3 Armstrong, D.B.: A Deductive Methode for Simulating Faults in Logic Circuits in: IEEE Trans. on Comp., Vol. C-21, No. 5, May 1972

4 Barzilai, Z. et al.: HSS- A High Speed Simulator, in: IEEE Transactions on Computer-Aided Design, Vol. 6, No. 4, July 1987, pp 601-617

5 Brglez, F.: A Fast Fault Grader: Analysis and Applications; Proc. International Test Conference, pp. 785-794, 1985

6 Brglez, F.et al.: Combinational Profiles of Sequential Benchmark Circuits in Proc. ISCAS'89, Portland, Oregon 1989, pp.1929-1934

7 Daehn, W.: A Switching Criterion for Hybrid ATPC; Proc. 1st European Test Conference, 1989, pp.26-32

8 Goel, P.: Test Generation Costs Analysis and l'rojections; Proc. 17th Design Automation Conference, June 1980, pp. 77-84

9 Harel, D.; Krishnamurthy, B.: Is there Hope for Linear Fault Simulation? Proc. 17th FTCS, 1987, pp. 28-33

10 Hong, S.J.: Fault Simulation Strategy for Combinational Logic Networks; Proc. 8th International Symposiun of Fault Tolerant Comp. (FTCS-8), 1978, pp. 96-99

11 Akers, S.B.et al.:Why is less information from logic simulation more useful in fault simulation? Proc. IEEE International Test Conference 1990, pp.786-800

12 Maamari, F.; Rajski J.: A Reconvergent Fanout Analysis for Efficient Exact Fault Simulation of Combinutional Circuits; Proc. FTCS-18, Tokyo 1988

13 Schulz, M.H.: Testmustergenerierung und Fehlersimulation in digitalen Schaltungen mit hoher Komplexität; Informatik-Fachberichte 173, Springer-Verlag 1988

14 Thompson, E.W.; Szygenda, S.A.: Parallel Fault Simulation; Computer, March 1975

15 Tarjan, R.E.: Finding Dominators in a directed graph; SIAM Journ. of Computing, Volume 3, 1974 pp. 62-89

16 Ulrich, E.G. and Baker, T.: The Concurrent Simulation of Nearly Identical Digital Networks; Proc. of 10th Design Automation Workshop,vol.6, pp.145-150, 1973

17 Waicukauski, J.A. et al.: Fault Simulation for Structured VLSI; VLSI Systems Design, Dec. 1985

18 Wunderñón. H.J.: Hochintegrierte Schaltungen: Prüfgerechter Entwurf und Test, Springer-Verlag, Berlin, Hcidelberg. New York, 1991 